\title{
Understanding the Evolving Cyberinfrastructure Needs of the Neuroscience Community
}

\author{
Amit Majumdar \\ San Diego Supercomputer Center \\ University of California San Diego \\ La Jolla, CA, USA \\ majumdar@sdsc.edu
}

\author{
Subhashini Sivagnanam \\ San Diego Supercomputer Center \\ University of California San Diego \\ La Jolla, CA, USA \\ sivagnan@sdsc.edu
}

\author{
Ted Carnevale \\ Department of Neurobiology \\ Yale School of Medicine \\ New Haven, CT, USA \\ ted.carnevale@yale.edu
}

\author{
Kenneth Yoshimoto \\ San Diego Supercomputer Center \\ University of California San Diego \\ La Jolla, CA, USA \\ kenneth@sdsc.edu
}

\begin{abstract}
In this paper, we first present a brief summary of the Neuroscience Gateway (NSG) which has been in operation since 2013. NSG is providing computational neuroscientists access to Extreme Science and Engineering Discovery Environment (XSEDE) high performance computing (HPC) resources. As a part of running the NSG we have interacted closely with the neuroscience community. This has given us the opportunity to receive input and feedback from the neuroscience researchers regarding their cyberinfrastructure needs. This is now more important given the context of the BRAIN (Brain Research through Advancing Innovative Neurotechnologies) Initiative which is a national initiative announced in 2013. Based on this interaction with the neuroscience community and the input we have received for the last three years, we analyze the comprehensive cyberinfrastructure needs of the neuroscience community in the second part of the paper.
\end{abstract}

\section{CCS Concepts}

- Software and its engineering $\rightarrow$ Software creation and management

\section{Keywords}

Neuroscience; Cyberinfrastructure; Collaborative environment.

\section{INTRODUCTION}

Past few decades have seen tremendous growth in computational neuroscience. This is noticeable from the emergence of new journals devoted to theoretical and computational neuroscience (e.g. Neural Computation, Journal of Computational Neuroscience)

Permission to make digital or hard copies of all or part of this work for personal or classroom use is granted without fee provided that copies are not made or distributed for profit or commercial advantage and that copies bear this notice and the full citation on the first page. Copyrights for components of this work owned by others than ACM must be honored. Abstracting with credit is permitted. To copy otherwise, or republish, to post on servers or to redistribute to lists, requires prior specific permission and/or a fee. Request permissions from Permissions@acm.org.

XSEDE16, July 17-21, 2016, ,

(C) 2016 ACM. ISBN 978-1-4503-4755-6/16/07 ...\$15.00

DOI: http://dx.doi.org/10.1145/2949550.2949657 as well as from the publication of modeling papers in the general neuroscience research literature. Similarly prominence of computational modeling in neuroscience is evident from proposals submitted to, and funded by, NSF, NIH and other organizations where modeling and computation played a significant role as a part of the proposed research. During the same time powerful computational neuronal simulation environments such as NEURON [1], GENESIS 3 [2], MOOSE [3], NEST [4], PyNN [5], Brian [6] and informatics tools for sharing and communicating computational models such as ModelDB [7, 8], Open Source Brain (OSB) [9], and Neuroscience Information Framework (NIF) [10] have evolved. These simulation environments and informatics tools are open source, mature and widely used in neuroscience research and many of them are developed using parallel programming framework suitable for current high performance computing (HPC) resources. As is well known by the past TeraGrid and the current XSEDE community, there has been significant development of cyberinfrastructure i.e. HPC, data resources, fast networks, workflows, and software during this time and it is enabling new science. The Neuroscience Gateway (NSG) [11] was introduced in 2013 and, as explained in the following section, it has been enabling the computational neuroscience community to easily and freely use HPC resources for bigger scale simulation of brain models, parameter sweep type simulations, and processing of large amount of brain imaging data.

The BRAIN (Brain Research through Advancing Innovative Neurotechnologies) [12] was announced by the White House in 2013 and is aimed at revolutionizing our understanding of the human brain. As a part of the BRAIN initiative, researchers will develop and apply innovative technologies and produce a new dynamic picture of the brain which will for the first time show how neurons and network of neuronal circuits interact in both space and time. This has direct implications in treating, curing and even preventing brain disorders and degenerative brain diseases. The BRAIN initiative involves dealing with huge amount of data and associated modeling and computation among experimental, computational and theoretical neuroscientists. As is clear, cyberinfrastructure (CI), including HPC, data resources, data processing, data storage, data sharing, software, and fast network will play a key role in making the BRAIN initiative successful. Although it is universally agreed upon that CI will be a very important part for the BRAIN initiative, it is not clear what ideal CI 
architecture is ultimately most useful for the neuroscience community such that it will provide all the compute and data resources as well provide a collaborative environment where computational and experimental neuroscientists can conduct research jointly.

In this paper we first describe briefly the current status of the NSG. Managing the operation of the NSG for over three years has allowed us to closely interact with neuroscientists and gain insight regarding the CI needs of the computational neuroscience community. We have collected input from large number of NSG users about their current and future CI needs. In addition we have run a survey where we have collected input from large number of neuroscientists regarding their CI needs. In the second half of the paper, we analyze the results of the survey and based on that make the case that a comprehensive cyberinfrastructure is needed for neuroscience research especially as research efforts evolve around the national BRAIN Initiative.

\section{NEUROSCIENCE GATEWAY}

\subsection{NSG Web Portal}

As stated in section 1, the past decades have seen significant development of both computational neuroscience and CI. During this time computational neuroscientists have initiated modeling and data processing projects that start out "small", and although their needs for resources grew, they were forced to stay "small" within the capacity of what can be accommodated in a desktop or small departmental or campus resources. This is often the case with projects that involve complex models (especially large scale neuronal networks) or complex protocols (often involving learning rules), or require high-dimensional optimization or parameter space exploration. Such projects have a tremendous need to use HPC, but only a very few neuroscientists have been able to perform simulations on large HPC resources $[13,14,15]$. There is a broad consensus that the wider computational neuroscience community needs access to HPC resources. Those who lack such access are at a significant disadvantage relative to very few who have it. This disparity is particularly telling in light of the fact that widely used simulators such as NEURON, GENESIS 3, MOOSE, NEST, and PyNN have been implemented on and released for HPC machines for several years now. Broadening the base by democratizing access for those who do not have first class computing at their institution was a strong motivator for developing the NSG.

Just like all the other science gateways, the NSG eliminates the administrative and technical barriers such as having to (i) write XSEDE allocation proposals every year, (ii) understand complex OS/software of always evolving HPC systems, (iii) install/test/benchmark neuroscience applications on various HPC resources, (iv) learn batch submission policies and scripts, and (v) deal with file systems and data retrieval. All of these issues are handled by the NSG team. And as a result NSG users can focus on neuroscience research by benefitting from (i) free and open access to XSEDE HPC resources, (ii) commonly used neuronal and image processing tools made available on HPC resources optimally, (iii) the simple web portal for uploading models and downloading results, (iv) the user interface for job query and notification of job completion, and (v) production quality user support. Neuroscience tool developers utilize NSG to test, benchmark and scale applications before they are released for the user community.

Since the NSG went into production in early 2013, NSG users have progressively utilized more HPC cycles every year. These HPC cycles are acquired via the peer reviewed XRAC allocation proposal system. Figure 1 shows the SUs used in years 2013, 2014, and 2015 in terms of Comet (the new HPC machine at SDSC) equivalent SUs by NSG users. And the SUs corresponding to year 2016 is the total amount acquired via XRAC for the year 2016 and currently (at the time of writing this paper in April, 2016) being used by the NSG users. We anticipate that, like previous years, all of the acquired SUs for 2016 will be used up by the NSG users by the year's end. As the plot shows, in 2013 NSG users utilized about 187,000 Comet equivalent SUs, in 2014 they used about 600,000 Comet equivalent SUs, in 2015 they used about 1,844,000 Comet equivalent SUs and in 2016 we acquired about 3,700,000 Comet equivalent SUs after favorable review of our XRAC proposal. For the years 2013, 2014, and 2015, NSG users used up the allocated SUs, and we had to acquire supplemental allocation to keep NSG operational. These show how NSG has been enthusiastically adopted by the neuroscience community and that there was a need for such a science gateway for the neuroscience community.

Figure 2 shows the maximum core count job submitted in each month since January 2013 and until May 2016. We see that the trend line of the maximum core count job has gone up towards 2000 cores and there has been 4000 core count jobs in the past. This is another indication that NSG users are utilizing larger scale simulations for brain models. The average job size of all the jobs (i.e. not just the maximum core count jobs as shown in Figure 2) during this period, from monthly average, is about 154 cores.

Some other factors that can be noted to show the impact that the NSG is having on the neuroscience community is that (i) the number of NSG users has grown steadily over the years to about 350 currently since it started in 2013, (ii) the community is requesting to add more neuroscience tools and especially image (e.g. MRI, fMRI, EEG) processing tools.

\subsection{REST Interface to NSG}

While operating NSG in the way described above we continued to interact with the neuroscience community closely and receive feedback from them regarding usage modes of NSG and possibilities of providing other means of accessing and using HPC resources.

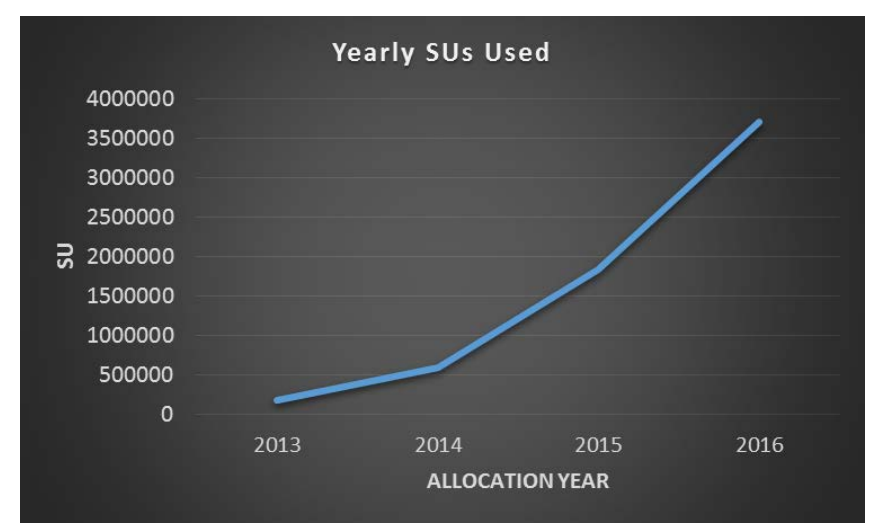

Figure 1. NSG SU Usage.

As a part of this continual interaction with the neuroscience community it became clear to us that users of informatics tools such as ModelDB, OSB, and NIF have become quite accustomed to the environment and workflow of those tools. Many of these neuroscience researchers are proficient in scripting and command line interaction and expressed the interest to have command line 
interaction capability such that they can work from the environment of the informatics tools.



Figure 2. Maximum Core Count of NSG Jobs.

In order to use the NSG via the originally intended web portal form, users must register and get individual accounts on NSG. Then they must log in to the NSG web site, upload their model source code, set NSG's job submission parameters, submit NSG jobs, and retrieve output results back to their local computers for further analysis. Those who want to work with a model from an informatics repository such as ModelDB face the additional step of downloading the model to their own computer before doing anything else. Although the majority of the users continued to use NSG via the web portal, we noticed the need for another set of users to have programmatic access to NSG.

Jointly with the neuroscience community we realized that the solution to this problem is to create a set of web services for the NSG and which will provide another way of accessing NSG (and associated HPC resources) other than the existing browser-based workflows. This set of web services will expose the capabilities of the NSG for access via publicly available application programmer interfaces (API). We have implemented this using the REpresentational State Transfer (REST) API [16] and it is called the NSG-R. Due to space limitation we do not describe the REST implementation in detail in this paper. NSG-R web services ensure that researchers can readily access HPC with a low overhead and in the context of their current workflows. This enables researchers to utilize the client NSG-R web services to connect directly to the NSG, automatically initiate simulations on HPC resources and retrieve output results back to their own familiar working environment. All of these steps are handled transparently by the client side API or library and require minimum user interaction. The seamless functional integration between HPC resources, via NSG web services API, should have powerful and broad impact on the productive use of computational modeling in neuroscience research.

\subsection{Neuroscience Image Processing}

In 2013 NSG started out by providing purely computational neuroscience tools such as NEURON, GENESIS, PyNN, Brian, MOOSE and NEST. Over the last year and a half we noticed the trend that more and more neuroscientists who are dealing with processing of MRI, fMRI, EEG type imaging data have approached us and requested that we provide image processing software such as FreeSurfer [17] and other image processing pipelines (e.g. The Virtual Brain processing pipeline [18]) through the NSG. Both FreeSurfer and The Virtual Brain pipeline are now provided as processing tools within NSG. Associated with this image processing, there has also been request for the ability to store large amount of data, share data, search data, make data publicly available for the community, access data etc.

\section{COLLABORATIVE ENVIRONMENT 3.1 Survey}

As described in section 2, our close interaction with the neuroscience community allowed us to understand the usage modes and various CI needs of the neuroscience community. In addition to the programmatic access (NSG-R) need, we realized that data processing, data sharing and access to data were the next topic of interest for the neuroscience community. And associated with this comprehensive data CI environment they wanted to have closely coupled compute/HPC capability and all within the context a collaborative environment which would allow computational and experimental neuroscientists to collaborate seamlessly.

To understand these needs from the broader neuroscience community we have developed a survey. The goal of the survey was to understand how the neuroscience community might need and use a CI solution for data management, collaboration, and computation.

The survey collected detailed information regarding the size of the research team, types of research data dealt with, size of data (currently and how it may grow in the future), what storage and access mechanism are there and how that may change over time, what are the current data management limitations, what compute needs are there and how it may grow, importance and need of a collaborative data-compute environment etc.

The survey was announced to the broader neuroscience community via various outlets in late February, 2016 and since then has been filled in by 81 neuroscientists. Many of the respondents to the survey represent bigger computational, or imaging, or neuroscience community projects such as the EEGlab [19], Open Source Brain, NEST software, NEURON software, a connectome research team etc. And hence effectively these 80 survey results represent a lot larger group of computational and experimental neuroscientists involved in collaborative research.

\subsection{Survey Results}

In this section we present some of the results from the survey. It should be noted that for the results shown in all the figures the respondents only had the option to choose one answer and hence the results would add up to $100 \%$.



Figure 3. Response to How Soon a Collaborative CI Would be Used by Neuroscience Researchers. 




Figure 4. Importance of a Collaborative Data Sharing Environment.

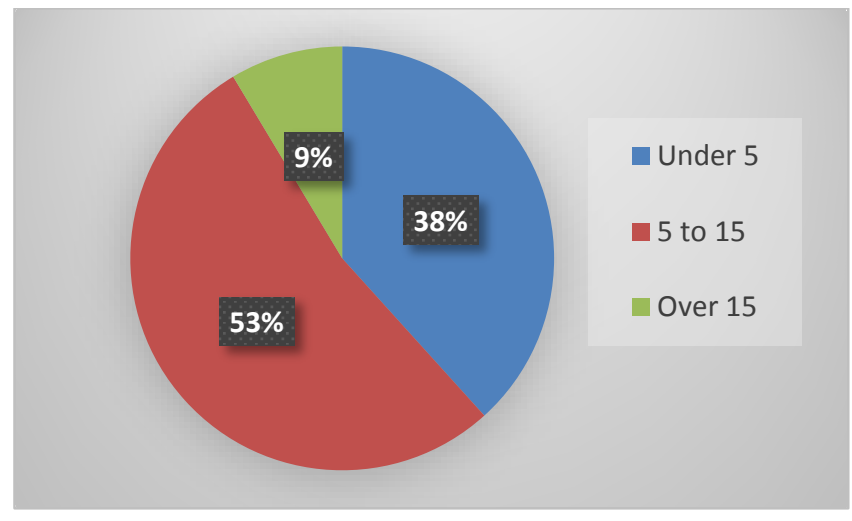

Figure 5. Size of Respondent's Neuroscience Research Team.



Figure 6. Professional Status of Respondents.

It should be noted that for results shown in all the tables, the respondents could choose multiple answers and hence the combined results are more than $100 \%$.
Table 1. Respondent's research data type

\begin{tabular}{|c|c|}
\hline Types of Data & Percentage \\
\hline Experimental & 77.8 \\
\hline Data from simulations & 85.2 \\
\hline Imaging data & 33.3 \\
\hline Text based survey data & 6.2 \\
\hline HIPAA compliant data & 7.4 \\
\hline Social network data & 0.0 \\
\hline Data collected in the field & 8.6 \\
\hline De-identified noncompliant data & 14.8 \\
\hline Sensor network data & 1.2 \\
\hline Other & 0.0 \\
\hline
\end{tabular}



Figure 7. Size of Current Research Data.

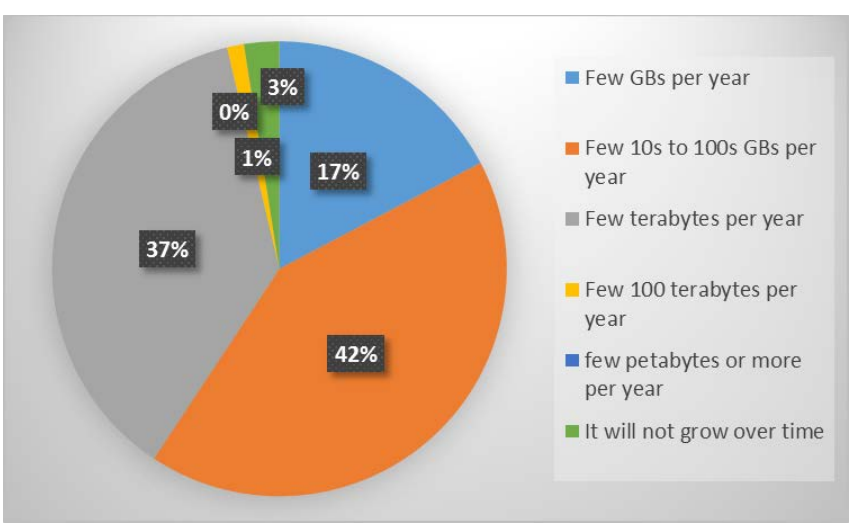

Figure 8. Expected Future Growth Rate of Research Data. 
Table 2. Mechanism of storing research data

\begin{tabular}{|c|c|}
\hline Mechanism & Percentage \\
\hline Personal lap-/desk-top & 65.4 \\
\hline Campus/institution storage & 58 \\
\hline External hard drive & 51.9 \\
\hline Cloud storage & 28.4 \\
\hline Other & 12 \\
\hline
\end{tabular}

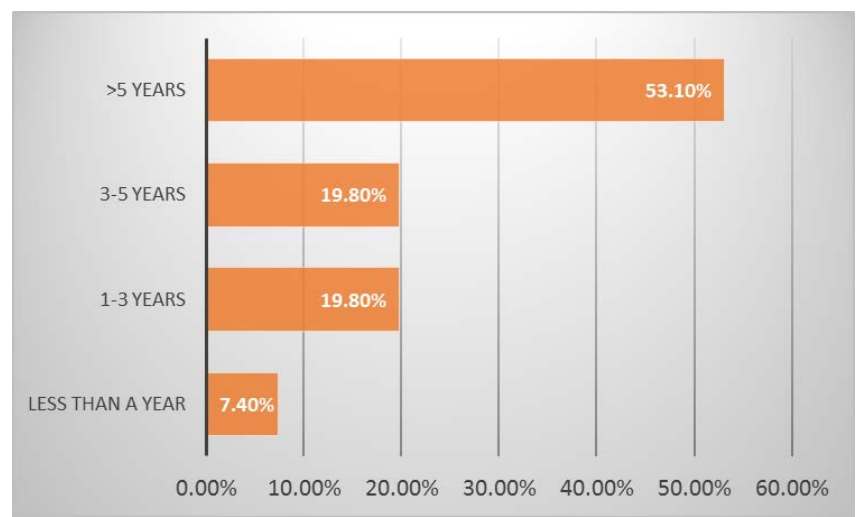

Figure 9. Expected Storage Time Length of Raw Data.

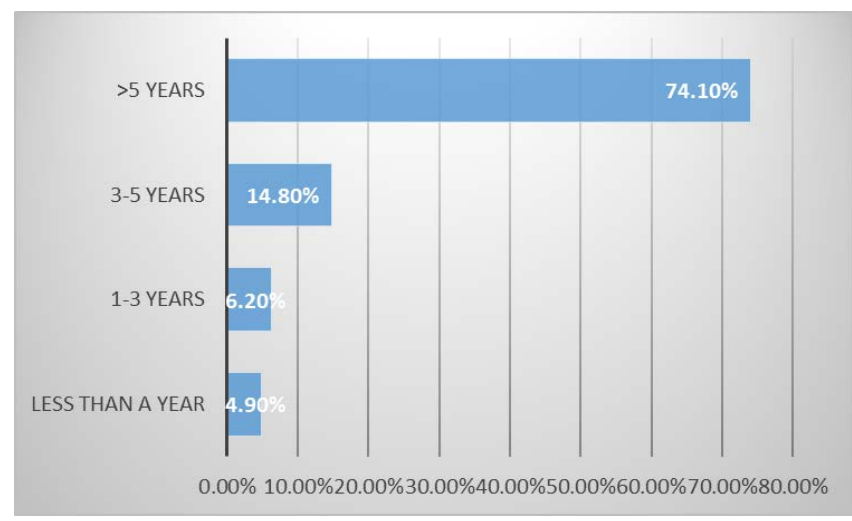

Figure 10. Expected Storage Time Length of Final Data.
Table 3. Pattern of data sharing

\begin{tabular}{|c|c|}
\hline Pattern & Percentage \\
\hline Not shared & 18.5 \\
\hline Shared with immediate collaborators & 82.7 \\
\hline Shared with collaborators in the country & 17.3 \\
\hline Shared with collaborators internationally & 44.4 \\
\hline Data is made publicly available & 34.6 \\
\hline Other & 12 \\
\hline
\end{tabular}

Table 4. Data sharing limitations

\begin{tabular}{|c|c|}
\hline Limitations & Percentage \\
\hline $\begin{array}{c}\text { No mechanism for sharing compliant (e.g. } \\
\text { HIPAA) data }\end{array}$ & 17.3 \\
\hline $\begin{array}{c}\text { Personal choice not to share data } \\
\text { and will then share with others }\end{array}$ & 3.7 \\
\hline $\begin{array}{c}\text { Nont mechanism for selective sharing (e.g. } \\
\text { select group) }\end{array}$ & 19.8 \\
\hline No limitations & 43.2 \\
\hline Other & 11.9 \\
\hline
\end{tabular}

Table 5. Current scope of data sharing

\begin{tabular}{|c|c|}
\hline Scope & Percentage \\
\hline Snail mail used to send hard disks & 14.8 \\
\hline Data sent via email & 46.9 \\
\hline Cloud or remote storage used to share data & 79 \\
\hline $\begin{array}{c}\text { Data transferred frequently (few times in } \\
\text { few months) }\end{array}$ & 19.8 \\
\hline $\begin{array}{c}\text { Data transferred less frequently (once/twice } \\
\text { a year) }\end{array}$ & 50.6 \\
\hline $\begin{array}{c}\text { Online resources used for data } \\
\text { sharing/access }\end{array}$ & 6.2 \\
\hline Other & \\
\hline
\end{tabular}


Table 6. Future choices for data sharing

\begin{tabular}{|c|c|}
\hline Scope & Percentage \\
\hline Want to share data via cloud & 63 \\
\hline Will pay for commercial cloud and HPC & 21 \\
\hline $\begin{array}{c}\text { Want to seamlessly move data from cloud } \\
\text { storage to compute and vice versa }\end{array}$ & 61.7 \\
\hline Need to sync cloud data with laptop/desktop & 49.4 \\
\hline $\begin{array}{c}\text { Not interested in cloud computing or } \\
\text { storage }\end{array}$ & 11.1 \\
\hline Other & 7.4 \\
\hline
\end{tabular}



Figure 11. Need for Search Capability on Data.

Table 7. HPC Needs

\begin{tabular}{|c|c|}
\hline Type & Percentage \\
\hline Few 1000 cores; $\sim 100$ teraflop & 79 \\
\hline$\sim 10,000-50,000$ cores; few petaflop & 39.5 \\
\hline$\sim 100,000+$ cores;; 10s-100s petaflop & 19.8 \\
\hline Exascale needed in the future & 13.6 \\
\hline HPC not needed & 6.2 \\
\hline Other & 3.7 \\
\hline
\end{tabular}

\section{SURVEY ANALYSIS}

Here we summarize the results of the survey. Figures 3 and 4 show that there is a strong need for a collaborative CI for the neuroscience community. Results of Table 1 show that most of the data are either experimental or computational, and a good amount of the data is coming from the neuroscience researchers involved in imaging research. This also implies that HPC would need to be available for simulations and processing of data and which is evident from Table 7. From Figures 7 and 8 we see that the collaborative environment needs to provide on the average $\sim$ few 10 s of TB of storage for each researcher and accommodate $\sim$ few TB of growth per year. We should mention that few researchers have stated that they need lot more ( PBs) of storage for their imaging data. Table 2 shows that most researchers are still storing data using local storage. Tables 3, 4, 5, and 6 shed light on various characteristics, issues and opportunities for designing a data sharing collaborative environment for neuroscientists.

This survey was generated based on insight gained from running the NSG for over three years and based on various input from the neuroscience community. The survey results can be considered as a first step towards designing a collaborative cyberinfrastructure for the neuroscience community.



Figure 12. NSG Portal Front Page.

Figure 12 shows the front page view of the NSG [20]. It should be noted the NSG is currently a science gateway that provides access to XSEDE HPC resources like most other XSEDE science gateways. NSG is not a data management (data sharing, data access, data publication etc.) environment and is also not a cyberinfrastructure environment where neuroscientists can collaborate, share data, access each other's neuronal models, develop models jointly etc. To develop a collaborative data/compute environment, based on the analysis of the survey results, one would need to investigate what existing data services can be utilized readily and/or need to be implemented to develop an integrated environment for neuroscientists.

\section{ACKNOWLEDGMENTS}

The research was supported by the US NSF awards DBI \#1458840, DBI \#1458495, DBI \#1146949, DBI \#1146830, ACI \#1339856 and UK BBSRC BB/N005236/1.

\section{REFERENCES}

[1] Carnevale, N. T., and Hines, L. M. 1993. The NEURON Book. Cambridge, UK: Cambridge University Press, 2006.

[2] Bower, J. M, and Beeman, D. The Book of GENESIS: Exploring Realistic Neural Models with the General Neural Simulation System. Second Edition, Springer-Verlag, New York (1998).

[3] http://moose.sourceforge.net/.

[4] Gewaltig M-O and Diesmann, M.NEST (Neural Simulation Tool) Scholarpedia, 2(4): 1430, 2007.

[5] Daviaon, A.P., Bruderle, D., Eppler, J.M., Kremkow, J., Muller, E., Pecevski D.A., Perrinet L., and Yger, P. PyNN: a common interface for neuronal network simulators. Front. Neuroinform.2:11, 2008

[6] Stimberg M., Goodman D.F.M, Benichoux, V., and Brette, R.Equation-oriented specification of neural models for simulations, Frontiers Neuroinf, 2014.

[7] http://senselab.med.yale.edu/modeldb/ 
[8] Hines ML, Morse T, Migliore M, Carnevale NT, Shepherd GM. ModelDB: A Database to Support Computational Neuroscience. Journal of Computational Neuroscience 2004 Jul-Aug;17(1):7-11.

[9] http://www.opensourcebrain.org

[10] http://neuinfo.org/

[11] S Sivagnanam, A Majumdar, K Yoshimoto, V Astakhov, A Bandrowski, M. E. Martone, and N. T. Carnevale. Introducing the Neuroscience Gateway, IWSG, volume 993 of CEUR Workshop Proceedings, CEUR-WS.org, 2013.

[12] http://www.braininitiative.nih.gov

[13] Ananthanarayanan R., Esser, S.K., Simon, H.D., and Modha, D.S. 2009. The cat is out of the bag: cortical simulations with $10^{9}$ neurons, $10^{13}$ synapses. In Proceedings of the Conference on High Performance Computing Networking, Storage and Analysis (SC '09). ACM, New York, NY, USA, Article 63, 12 pages. DOI=http://dx.doi.org/10.1145/1654059.1654124

[14] Markram, H. The Blue Brain Project. Nature Reviews Neuroscience 7, 153-160 (1, February 2006)
[15] Kumar, S., Heidelberger, P., Chen, D. and Hines, M. Optimization of applications with non-blocking neighborhood collectives via Multisends on the Blue Gene/P supercomputer. 24th IEEE International Parallel and Distributed Processing Symposium, in press, 2010.

[16] Fielding, R.T, and Taylor, R.N. Principled design of the modern Web architecture. In Proceedings of the 22nd international conference on Software engineering (ICSE '00). ACM, New York, NY, USA, 407-416, 2000, DOI $=10.1145 / 337180.337228$ http://doi.acm.org/10.1145/337180.337228

[17] http://freesurfer.net/

[18] M. Schirner, S. Rothmeier, V. K. Jirsa, A. R. McIntosh, P. Ritter, An Automated Pipeline for Constructing Personalized Virtual Brains from Multimodal Neuroimaging Data, NeuroImage, March 2015;

doi:10.1016/j.neuroimage.2015.03.055

[19] http://sccn.ucsd.edu/eeglab/index.php

[20] http://www.nsgportal.org 